VOL. $73(2006) \quad[479-479]$

\title{
Quantum superalgebras at roots of unity and topological invariants of three-manifolds
}

\author{
SACHA C. BLUMEN
}

The general method of Reshetikhin and Turaev is followed to develop topological invariants of closed, connected, orientable 3-manifolds from a new class of algebras called pseudo-modular Hopf algebras. Pseudo-modular Hopf algebras are a class of $\mathbb{Z}_{2}$-graded ribbon Hopf algebras that generalise the concept of a modular Hopf algebra.

The quantum superalgebra $U_{q}(\operatorname{osp}(1 \mid 2 n))$ over $\mathbb{C}$ is considered with $q$ a primitive $N^{\text {th }}$ root of unity for all integers $N \geq 3$. For such a $q$, a certain left ideal $\mathcal{I}$ of $U_{q}(\operatorname{osp}(1 \mid 2 n))$ is also a two-sided Hopf ideal, and the quotient algebra $U_{q}^{(N)}(\operatorname{osp}(1 \mid 2 n))=U_{q}(\operatorname{osp}(1 \mid 2 n)) / \mathcal{I}$ is a $\mathbb{Z}_{2}$-graded ribbon Hopf algebra.

For all $n$ and all $N \geq 3$, a finite collection of finite dimensional representations of $U_{q}^{(N)}(\operatorname{osp}(1 \mid 2 n))$ is defined. Each such representation of $U_{q}^{(N)}(\operatorname{osp}(1 \mid 2 n))$ is labelled by an integral dominant weight belonging to the truncated dominant Weyl chamber. Properties of these representations are considered: the quantum superdimension of each representation is calculated, each representation is shown to be self-dual, and more importantly, the decomposition of the tensor product of an arbitrary number of such representations is obtained for even $N$.

It is proved that the quotient algebra $U_{q}^{(N)}(\operatorname{osp}(1 \mid 2 n))$, together with the set of finite dimensional representations discussed above, form a pseudo-modular Hopf algebra when $N \geq 6$ is twice an odd number.

Using this pseudo-modular Hopf algebra, we construct a topological invariant of 3-manifolds. This invariant is shown to be different to the topological invariants of 3 -manifolds arising from quantum $s o(2 n+1)$ at roots of unity.

School of Mathematics and Statistics

University of Sydney

New South Wales 2006

Australia

e-mail: sachab@maths.usyd.edu.au

Received 9th January, 2006

Thesis submitted to the University of Sydney, April 2005. Degree approved, December 2005. Supervisor: Associate Professor RB Zhang.

Copyright Clearance Centre, Inc. Serial-fee code: 0004-9727/06 \$A2.00+0.00. 